\title{
CASTOR: Centauro And Strange Object Research in nucleus-nucleus collisions at LHC
}

\author{
Ewa Gtadysz-Dziaduś for the CASTOR group
}

\author{
A L S Angelis ${ }^{1}$, X Aslanoglou ${ }^{2}, \mathrm{~J}$ Bartke $^{3}, \mathrm{~K} \mathrm{Chileev}^{4}$, \\ E Gładysz-Dziaduś ${ }^{3}{ }^{*}$, M Golubeva $^{4}$, F Guber ${ }^{4}, \mathbf{T}$ Karavitcheva ${ }^{4}$, \\ Y V Kharlov ${ }^{5}$, A B Kurepin ${ }^{4}$, G Mavromanolakis ${ }^{1}$, A D Panagiotou ${ }^{1}$, \\ S A Sadovsky ${ }^{5}$ V V Tiflov ${ }^{4}$, and Z Włodarczyk ${ }^{6}$ \\ ${ }^{1}$ Nuclear and Particle Physics Division, University of Athens, Athens, Greece. \\ ${ }^{2}$ Department of Physics, the University of Ioannina, Ioannina, Greece. \\ ${ }^{3}$ Institute of Nuclear Physics, Cracow, Poland. \\ ${ }^{4}$ Institute for Nuclear Research, Moscow, Russia. \\ ${ }^{5}$ Institute for High Energy Physics, Protvino, Russia. \\ ${ }^{6}$ Institute of Physics, Pedagogical University, Kielce, Poland.
}

\begin{abstract}
.
We describe the CASTOR detector designed to probe the very forward, baryon-rich rapidity region in nucleus-nucleus collisions at the LHC. We present a phenomenological model describing the formation of a QGP fireball in high baryochemical potential environment, and its subsequent decay into baryons and possibly strangelets. The model explains the Centauro events observed in cosmic rays and the long-penetrating component frequently accompanying them, and makes predictions for the LHC. Simulations of Centauro-type events by means of our Monte-Carlo event generator CNGEN were done. To study the response of the apparatus to new effects, different exotic species (DCC clusters, Centauros, strangelets and so-called mixed events produced by baryons and strangelets being the remnants of the Centauro fireball explosion) were passed through the deep calorimeter. The energy deposition pattern in the calorimeter appears to be a new clear signature of the QGP state.
\end{abstract}

\section{Introduction}

The motivation to study the very forward phase space in nucleus-nucleus collisions at the LHC stems from the potentially very rich field of new phenomena which can be produced in an environment of very high baryochemical potential. The study of this baryon-dense region in the laboratory will provide important information for the understanding of a Quark-Gluon Plasma (QGP) state at relatively low temperatures, with different properties from the one in the higher temperature baryonfree region around mid-rapidity, which could exist in the core of neutron stars. Although there are serious technical difficulties in doing calculations for a high baryochemical potential environment, many physicists agree that a lot of interesting phenomena predicted by theory and/or announced by experiments should appear in this region.

In particular, some theoretical considerations suggest [1] that the phase diagram features a critical endpoint $\mathrm{E}\left(T_{E} \simeq 160 \mathrm{MeV}\right.$ and $\left.\mu_{E} \simeq 725 \mathrm{MeV}\right)$ at which the line of the first order phase transition $\left(\mu>\mu_{E}\right.$ and $\left.T<T_{E}\right)$ ends. At this point the phase transition becomes of second order and long wavelength fluctuations appear. Passing close enough to this critical endpoint should have characteristic experimental consequences. Since one can miss the critical point on either of two sides a nonmonotonic dependence of the control parameters should be expected.

Other theoretical ideas attracting a lot of attention are: colour superconductive state at finite baryon density [2] or skyrmions [3] - coherent states of baryons possibly produced by a DCC. Also strangelets, droplets of strange quark matter are predicted to be formed from the Quark Gluon Plasma, predominantly in a high baryochemical potential environment [4]. Heavy flavour [5] and Super Heavy Particles [6] production is expected to dominate in the forward rapidity region.

It is especially important to note that high energy cosmic ray interactions show the existence of the wide spectrum of exotic events (Centauros, Mini-Centauros, Chirons, Geminions, Halo-type events etc. $[7,8]$ ) observed at forward rapidities. These so-called Centauro species reveal many surprising features, such as: abnormal hadron dominance, transverse momentum of produced particles much higher than that observed in "normal" interactions, the existence of mini-clusters etc.. Besides that they are very frequently connected with the so-called long-flying (penetrating) component [8]-[11].

* Corresponding author (ewa.gladysz@ifj.edu.pl)

Further information and complete bibliography at http://home.cern.ch/angelis/castor/Welcome.html 
These anomalies are observed at energies above $\sim 10^{15} \mathrm{eV}$ and are not rare occurence but they manifest themselves at about $5 \%$ level. It is widely believed that Centauro related phenomena could not be due to any kind of statistical fluctuation in the hadronic content of normal events and they have until now defied all attempts at explanation in terms of conventional physics [12]. Instead many unconventional models have been proposed. Some of them (e.g. [13]) assume that exotic objects of unknown origin are present in the primary cosmic ray spectrum and they are seen as Centauros during their penetration through the atmosphere. Others assume that exotic fireballs are produced in extremely high energy hadron-hadron (e.g. [14]) or nucleus-nucleus (e.g. [15, 16]) interactions. Other unconventional attempts, as for example a DCC scenario [17] or the color-sextet quark model [18], based on Pomeron physics in QCD were also developed. The widespread opinion that the likely mechanism for Centauro production is the formation of a quark-gluon plasma was incorporated in a lot of proposed models. But only the model of the strange quark-matter fireball $[15,16,19]$ explains simultaneously both the main features of the Centauro-like events and the strongly penetrating component accompanying them. Both the experimental characteristics of Centaurorelated species and the model predictions indicate the forward rapidity region as the most favourable place for production and detection of such anomalous phenomena.

The LHC will be the first accelerator to effectively probe the very high energy cosmic ray domain, where cosmic ray experiments have detected numerous very unusual events. These events may be produced and studied at the LHC in controlled conditions. Majority of the present and future nucleusnucleus experiments concern the exploration of the baryon-free region and "midrapidity physics". Already several years ago we announced the necessity to investigate the forward rapidity region in future heavy ion experiments [20]. A small collaboration has been formed and the CASTOR detector, a unique experimental design to probe the very forward rapidity region in nucleus-nucleus collisions at the LHC and to complement the CERN heavy ion physics program pursued essentially in the baryon-free midrapidity region, has been proposed [21]. In order to illustrate the detector's sensitivity to new effects we have done simulations of Centauro-type events by means of our Monte-Carlo event generator CNGEN [22]. The simulated Centauro events have characteristics manifestly different from those predicted by "classical" (e.g. HIJING) generators [8, 23]. The different exotic species were also followed through the deep calorimeter by means of modified GEANT 3.21 [8, 23, 24]. We simulated transition curves produced in the CASTOR calorimeter by: DCC clusters (both neutral and charged), Centauros, strangelets (both stable and unstable) and so-called mixed events produced by baryons and strangelets as the remnants of the Centauro fireball explosion. To study the sensitivity of the calorimeter to abnormally penetrating objects a neural network technique was also developed [25]. Different exotic phenomena give different energy deposition patterns and can be well distinguished from the usual events as well as from one another.

\section{Centauro-like phenomena in cosmic ray experiments}

Centauro related phenomena were discovered and have been analysed in emulsion chamber experiments investigating cosmic ray interactions at the high mountain laboratories at Mt. Chacaltaya (5200 m above see level ) and Pamirs ( 4300 or $4900 \mathrm{~m}$ above sea level). Both the experimental aspects and the model explanations have been presented in the recent review [8].

The experimental results show that hadron-rich families constitute more than $20 \%$ of the whole statistics [11] as is illustrated in Fig. 1. This conclusion has been drawn from the analysis of the unbiased sample of 429 families from Chacaltaya (open circles), 173 from the Pamir-Joint chambers and 135 from a part of the Pamir chambers of $500 \mathrm{~m}^{2} \mathrm{yr}$ (closed circles) with total visible energy greater than $100 \mathrm{TeV}$. A scatter diagram of $N_{h}$ vs. $Q_{h}$ is shown, where $N_{h}$ denotes the number of

hadrons in a family with visible energy greater than $4 \mathrm{TeV}$, and $Q_{h}=\Sigma E_{h}^{(\gamma)} /\left(\Sigma E_{(\gamma)}+\Sigma E_{h}^{\gamma}\right)$ is the fraction of the total visible energy carried by these hadrons.

The experimental data reveal the existence of several types Centauro species such as:

(i) Centauros of original type, 

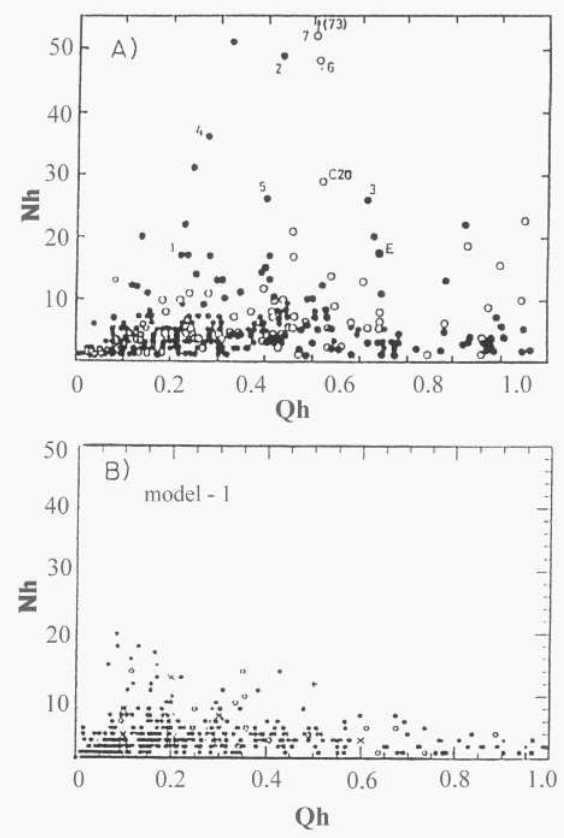

Figure 1. (A) $N_{h}-Q_{h}$ diagram of families detected in Pamir, Chacaltaya and Pamir-Joint chambers, (B) The same for the simulated families. Different marks signify the different primary cosmic-ray nuclei: $(\bullet)$ proton, (०) $\alpha,(\diamond) \mathrm{CNO},(\times)$ heavy, (+) Fe, [11].

(ii) Mini-Centauros,

(iii) Chirons,

(iv) Geminions.

They are all characterized by:

- Abnormal hadron dominance (both in multiplicity and in energy content). For "classical" Chacaltaya Centauros hadron multiplicity $<N_{h}>\sim 75$ in comparison to electron/gamma multiplicity $<N_{\gamma}>\sim 0$.

- Low total (hadron) multiplicity, in comparison with that expected for nucleus-nucleus collisions at that energy range.

- Transverse momentum of produced particles much higher than that observed in "normal" interactions $\left(p_{T} \approx 1.7 \mathrm{GeV} / \mathrm{c}\right.$ for Centauros and $10-15 \mathrm{GeV} / \mathrm{c}$ for Chirons, assuming a gamma inelasticity coefficient $K_{\gamma} \approx 0.2$ ).

- Psedorapidity distributions consistent with a nearly Gaussian distribution and experimental characteristics supporting the formation and subsequent isotropic decay of a fireball with hadron multiplicity $N_{h} \sim 100$ and mass $M_{f b} \sim 180 \mathrm{GeV}$ for Centauros and Chirons, and $N_{h} \sim 15$ and $M_{f b} \sim 35 \mathrm{GeV}$ for Mini-Centauros.

Besides that they are very frequently connected with the so-called long-flying (penetrating) component. In fact, the strongly penetrating component has two aspects. At first, it has been observed in the apparatus in the form of strongly penetrating single cascades, clusters of showers or the so called "halo". This phenomenon manifests itself by the characteristic energy pattern revealed in shower development in the deep chambers (calorimeters) indicating the slow attenuation and many maxima structure. The second aspect is connected with the anomalously strong penetrability of some objects in the passage through the atmosphere. In principle, both aspects can be connected one to each other and could be different manifestations of the same phenomenon.

Anomalous cascade transition curves have been firstly noticed during the study of Chiron-type families, where the simultaneous appearance of the unusual hadronic component with the short interaction mean free path, as small as $\sim 1 / 3-1 / 2$ of the nucleon geometrical collision mean free path has been observed. Subsequently, the strongly penetrating component has been encountered 
also in other kinds of events [8]-[11]. Some of them penetrate through the whole apparatus without noticeable attenuation, sometimes even indicating a tendency to grow. The most spectacular examples are two exotic cascades detected in the Centauro-like event $\mathrm{C}-\mathrm{K}$ [10] found in the homogenous type deep lead chamber. They were observed not far from the energy weighted centre of the family, at very close distance one to each other. Both cascades demonstrated a multicore structure and unexpectedly long range and many maxima character. After passing through a very thick layer of lead, they escaped through the bottom of the chamber. The longer cascade, shown in Fig. 2 penetrated more than 109

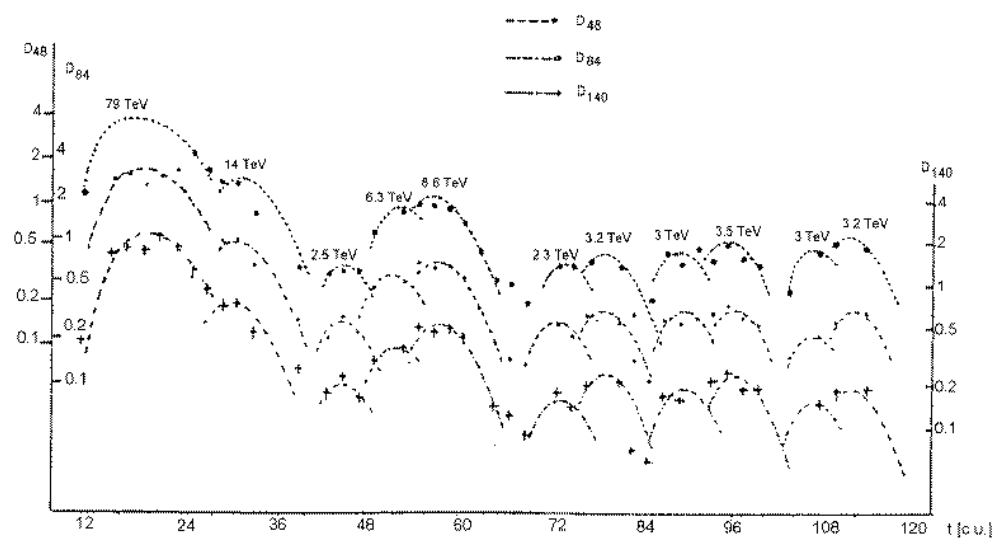

Figure 2. Transition curves in X-ray film darkness D (measured in three diaphragms of a radius $\mathrm{R}=48,84$ and $140 \mu$ ) for cascade no. 197.08. Energy (in TeV units) liberated into the soft component is indicated at each hump (averaged over three estimated values) [10].

cascade units and 11 maxima appeared along its transition curve. The average distance between the neighbouring humps is about two times shorter than theoretically expected for conventional hadronic cascades. Recent simulations, assuming four models of hadron-nucleus interactions (VENUS 4.12, QGSJET, HDPM and modified UA5, all widely used as standard models) confirmed the unusual character of long-penetrating cascades [12].

\section{Phenomenological model}

According to the model developed in $[15,16]$ Centauro arises through the hadronization of a QGP fireball of very high baryochemical potential $\left(\mu_{\mathrm{b}}>>\mathrm{m}_{\mathrm{n}}\right)$, produced in nucleus-nucleus collisions in the upper atmosphere. In this model the QGP fireball initially consists of $u$ and d quarks and gluons. The very high baryochemical potential inhibits the creation of u $\bar{u}$ and d $\bar{d}$ quark pairs, resulting

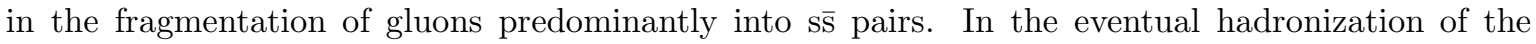
fireball this leads to the strong suppression of pions and hence of photons. As the fireball evolves strangeness distillation through emission of kaons [4] transforms the initial quark matter fireball into an increasingly strange quark matter state. The stabilizing effects of the strangeness excess prolong the lifetime of the fireball, enabling it to reach mountain-top altitudes [26]. In the subsequent decay and hadronization of this state separation of strangeness can lead to the formation of non-strange baryons, hyperons and/or strangelets. The main stages of the time development of the Centauro fireball are shown in Fig. 3.

The hypothesis that strangelets can be identified as the strongly penetrating particles frequently seen accompanying hadron-rich cosmic ray events has been made and checked $[16,19]$.

Our simulations show that the transition curves produced by strangelets during their passage through the chamber resemble the experimentally detected ones. As an example three cascade curves produced by unstable, metastable and unstable strangelets respectively are shown in Fig. 4. The longrange cascades observed in the thick homogenous lead/emulsion chambers could be the result of a 


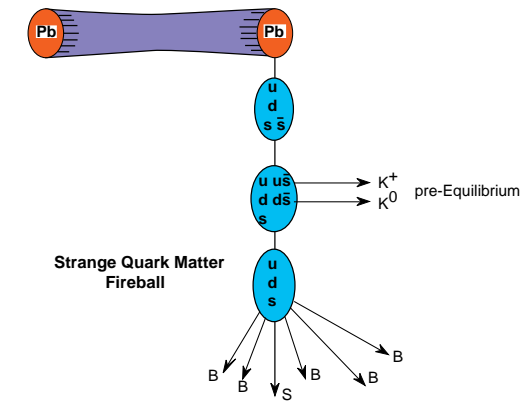

Figure 3. Centauro fireball evolution scheme.

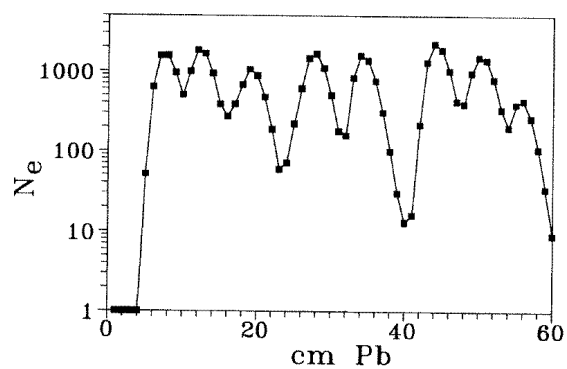

Unstable strangelet decaying into a bundle of 7 neutrons $\left(E_{n} \simeq E_{\text {str }} / A_{\text {str }} \simeq 200 \mathrm{TeV}\right)$.

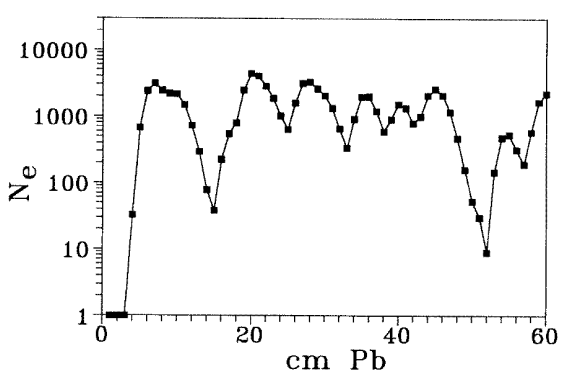

Long-lived strangelet $\left(A_{\text {str }}=15, \mu_{q}=600 \mathrm{MeV}\right)$.

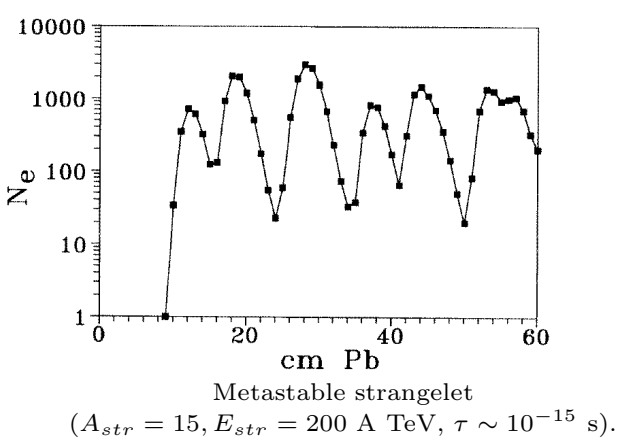

Figure 4. Examples of simulated transition curves recorded in the lead chamber and produced by various strangelets. Numbers of electrons $N_{e}$ are counted within the radius of $50 \mu \mathrm{m}$.

strangelet penetration through the apparatus. Their strong penetrating power can be connected both with the small interaction cross section (in comparison with nucleus of comparable A) of the strangelet and with the big concentration of its energy in a narrow region of phase space. This energy could be liberated into conventional particle production in many consecutive evaporation or interaction acts. In this way numerous hadron-rich families accompanied by highly penetrating cascades, clusters or halo could be explained by assuming the same mechanism of the formation of a strange quark matter fireball and its successive decay into predominantly baryons and strangelet(s).

Therefore, in this model both the basic characteristics of cosmic ray Centauro (small multiplicities and extreme imbalance of hadronic to electromagnetic content) and the strongly penetrating component are naturally explained. The model is used to make predictions for Centauro and strangelet formation in $\mathrm{Pb}+\mathrm{Pb}$ collisions at the LHC. In table 1 we compare characteristics of Centauro and strongly penetrating objects ("strangelets"), either experimentally observed or calculated within the context of the model, for cosmic ray interactions and for $\mathrm{Pb}+\mathrm{Pb}$ interactions at the LHC.

\section{Strange Quark Matter - a possible state of matter}

As the search for strongly penetrating species which could be produced by strangelets is one of the aims of the CASTOR detector, some questions concerning their possible production and consequences of their existence should be considered. It is especially important in view of the recent debate [27][31] on the possibility of a catastrophic scenario initiated by strangelets formed in the future collider experiments.

The strange quark matter (SQM) is a matter with strangeness per baryon of the order of unity, containing a comparable fraction of up, down and strange quarks. The existence of stable SQM 
Table 1. Average characteristic quantities of modeled Centauro and Strangelets produced in Cosmic Rays and expected at the LHC.

\begin{tabular}{ccc}
\hline Centauro & Cosmic Rays & LHC \\
\hline Interaction & "Fe $+\mathrm{N} "$ & $\mathrm{~Pb}+\mathrm{Pb}$ \\
$\sqrt{s}$ & $\geq 6.76 \mathrm{TeV}$ & $1148 \mathrm{TeV}$ \\
Fireball mass & $\geq 180 \mathrm{GeV}$ & $\sim 500 \mathrm{GeV}$ \\
Projectile rapidity $y_{p r o j}$ & $\geq 11$ & 8.67 \\
Lorentz factor $\gamma$ & $\geq 10^{4}$ & $\simeq 300$ \\
Centauro pseudorapidity $\eta_{\text {cent }}$ & 9.9 & $\simeq 5.6$ \\
$\Delta \eta_{\text {cent }}$ & 1 & $\simeq 0.8$ \\
$<p_{T}>$ & $1.75 \mathrm{GeV}$ & $1.75 \mathrm{GeV}(*)$ \\
Lifetime & $10^{-9} \mathrm{~s}$ & $10^{-9} \mathrm{~s}(*)$ \\
Decay probability & $(\mathrm{x} \geq 10 \mathrm{~km}) 10 \%$ & $(\mathrm{x} \leq 1 \mathrm{~m}) 1 \%$ \\
Strangeness & 14 & $60-80$ \\
$f_{s}$ (S/A) & $\simeq 0.1-0.4$ & $0.30-0.45$ \\
Z/A & $\simeq 0.3-0.4$ & $\simeq 0.2$ \\
Event rate & $\simeq 1 \%$ & $\simeq 0.1 \%$ \\
\hline "Strangelet" & Cosmic Rays & LHC \\
\hline Mass & $\simeq 7-15 \mathrm{GeV}$ & $10-80 \mathrm{GeV}$ \\
$f_{s}$ & $\simeq 1$ & $\simeq 1$ \\
\hline Strangelet pseudorapidity $\eta_{\text {str }}$ & $\eta_{\text {cent }}+1.2$ & $\eta_{\text {cent }}+1.2$ \\
\hline (*) &
\end{tabular}

(*) assumed

was postulated by E. Witten [32]. Introduction of a third flavour creates an additional Fermi well and thus reduces the energy relative to a two-flavour system what can thereby making the system stable. The anticipated mass range for this kind of matter lies anywhere between the masses of light nuclei and neutron stars $A \simeq 10^{57}$. The latter are called strange stars. Strange matter as part of cosmic radiation is sometimes reffered to as strange quark nuggets or nuclearities. Smaller amounts of strange quark matter are usually called droplets of strange quark matter, or simply strangelets. Its existence, however, is purely an experimental question since computations in QCD cannot come close to answering this enormously important conjecture. SQM could have important cosmological consequences for today's universe which arise from the possibility that the early universe might have undergone a first-order phase transition from SQM to the nuclear matter. Strangelets left over from that era could also account for the cosmological dark matter problem.

The properties of some forms of hypothetical strange matter, as small lumps of strange quark matter (strangelets) or hyperon matter (metastable multihypernuclear objects MEMO's) have been widely discussed with special emphasis on their relevance to the present and future heavy ion experiments. Different aspects of strange quark matter physics, as for example the stability question are described in the reviews [33]. Contrary to normal nuclei, SQM stability increases with A and the threshold of its stability is close to $A_{\text {crit }} \sim 300$. But also quite small strangelets might gain stability due to shell effects. However, due to the lack of theoretical constraints on bag model parameters and difficulties in calculating color magnetic interactions and finite size effects, experiments are necessary to answer the question of the stability of strangelets.

Strangelets may arise from various scenarios; they could be formed in high energy nuclear collisions or they might be of cosmological origin, as remnants of the cosmic QCD phase transition. Collisions of strange stars could also lead to the formation of strangelets which could contribute to the cosmic ray flux. E. Witten [32] suggested the possibility of production of small lumps of SQM, in today's universe by quark(neutron) stars, in the process of their conversion to more stable SQM stars. Several types of models are applied to describe the strangelet production in heavy ion collisions. They can be classified into two categories, namely strangelet production by coalescense of hyperons or by production following a creation of quark-gluon plasma. In a very popular coalescense model, an ensemble of quarks, which 
are products of nucleus-nucleus collisions, form a composite state which fuses to form a strangelet [34]. Formation of quark-gluon plasma is not needed in this scenario, as hyperons coalesce during the late stage of the collision forming a doorway state for strangelet production. Such a scenario favours the production of low mass strangelets ( $A \geq 10$ is rather unlikely) in the midrapidity region. Thermal models assume that chemical and thermal equilibrium are achieved prior to final particle production. Coalescense and thermal models usually predict lower strangelet cross section than models postulating a QGP as an intermediate state in a strangelet formation. The strangelet distillation mechanism provides a possibility for producing more stable large strangelets since the QGP would lose energy by meson emission, possibly resulting in a strangelet of approximately the same A as the QGP droplet.

The present status of strange quark matter searches, both in cosmic rays and in accelerator experiments is presented by R. Klingenberg in his recent review [35]. The experimental situation concerning the existence of SQM is quite intriguing.

Many accelerator experiments are looking for strange quark matter in heavy ion collisions. They are mostly based on such discerning properties of strangelets as an unusual charge to mass ratio $(Z / A \ll 1)$. The strange counterparts of ordinary nuclei are searched for in high-energy collisions at Brookhaven and at CERN. To date no experiment has published results indicating a clear positive signal for strangelets, although some candidates have been announced [36]. Accelerator experiments are able to set upper limits on the existence of strangelets in the range of sensitivity of the experiments but they are not able to answer the question concerning their existence. There are several definite reasons for this. The main ones are that the experiments are sensitive only to metastable strangelets with proper lifetimes greater than $\sim 5 \cdot 10^{-8} \mathrm{~s}$ and that they look for strangelets in the midrapidity region. Production limits obtained in these experiments are strongly dependent upon the production model assumed for lumps of strange quark matter.

Searches for SQM have been made also on terrestrial matter, cosmic rays and astrophysical objects. The searches resulted in low limits for strangelets in terrestrial matter but on the other hand two seismic events with the properties of the passage of SQM nuggets through the Earth have been reported recently [37]. Morever, a lot of cosmic ray anomalies could be understood by assuming the presence of strangelets in cosmic ray spectrum. These, reviewed in $[8,38]$ are:

(i) Massive and relatively low charged objects, i.e.:

- Two anomalous events, with value of charge $Z \simeq 14$ and of mass number $A \simeq 350$ and $\simeq 450$ observed in primary cosmic rays by a balloon counter experiment [39];

- The so-called Price's event [40] with $\mathrm{Z} \simeq 46$ and $\mathrm{A}>1000$;

- The so-called Exotic Track event with $\mathrm{Z} \simeq 20$ and $\mathrm{A} \simeq 460$ [41]. It was observed in a balloon-borne emulsion chamber exposed to cosmic rays at the atmospheric depth of only $11.7 \mathrm{~g} / \mathrm{cm}^{2}$ at zenith angle of $87.4^{0}$. This means that the projectile that caused that event traversed $\sim 200 \mathrm{~g} / \mathrm{cm}^{2}$ of the atmosphere.

(ii) Muon bundles from CosmoLEP [42].

(iii) Delayed neutrons [43] observed in large Extensive Air Showers by the neutron monitor working in conjunction with EAS instalation "Hadron".

(iv) CENTAURO-related phenomena.

As well as Centauro-like events as the strongly penetrating component are postulated to be the signs of strangelets passage through the matter. Some explanations assume that strangelets are a component of primary cosmic rays [44], while others assume that they are produced in collisions of cosmic ray nuclei in the atmosphere $[16,19]$.

There are some speculations $[27,28,29]$ that under some conditions the small metastable SQM drops (produced for instance in heavy ion collisions) could be "rapidly grown" to a larger stable size, feeding in neutrons or light nuclei and releasing energy in photons. For such behaviour numerous conditions should be fulfilled. First of all strangelets must have rather low velocity (must be produced close to the midrapidity in collider experiments) and a negative charge. A number of reasons have been put forward, theoretical as well as experimental, why such a scenario is exceedingly unlikely [28]-[31] and 
it is argued that experiments at RHIC and LHC do not represent a threat to our planet. The main arguments are:

- Large safety factors derived from the survival of the Moon and the observed rate of supernovae $[28,29]$;

- Phenomena indicating the appearance of strangelets in cosmic rays;

- Much higher probability of strangelet production in baryon-rich fragmentation regions than at midrapidity;

- Predicted positive charge of strangelets [30, 31].

The strongest argument seems to be the existence of the aformentioned phenomena which could be the manifestation of strangelets in cosmic rays. Among theoretical arguments the prediction of a positive electric charge for strangelets seems to be especially convincing one. Strange matter would have been neutral, if the ground state composition consisted of equal numbers of quarks of three flavours, which is the most favoured state. Actually, however, the $s$ quark is heavier than the other two flavours and so their number is slightly less than that of the $u$ and $d$ quarks, as a result of which it has a small positive charge. J. Madsen showed [30] that, in fact, stable SQM may be negatively charged in bulk, however, the reduction in strange quark occupation in the surface layer, which is responsible for surface tension, causes the intermediate mass strangelets to be always positively charged. Morever, in his more recent work [31] he showed that strange-quark matter in a color-flavour locked state is bound stronger than "ordinary" strange quark matter. This increases the probability of formation of metastable or even absolutely stable strangelets and leads to a stronger reduction in number of negatively charged s-quarks in the surface layer. A total positive quark charge of color-flavour locked strangelets is expected to be proportional to the surface area or $\mathrm{A}^{2 / 3}$. The effect is large enough to rule out a potential disaster scenario.

\section{The CASTOR detector}

CASTOR will probe the very forward rapidity region in nucleus-nucleus collisions at the LHC and search for new effects. The main part of the detector will be a deep calorimeter. Other parts, as for example photon and hadron multiplicity detectors are also considered. The calorimeter [21] (Fig. 5) will be azimuthally divided into 8 octants and longitudinally segmented into layers, each layer consisting of a tugsten absorber plate followed by a number of quartz fibre planes. The signal is the Cherenkov light produced in the fibres as they are traversed by relativistic charged particles in the showers. The layers are inclined at $45^{\circ}$ relative to the impinging particles in order to maximize the Cherenkov light yield. The light propagates along the fibres to the outer edge of the calorimeter where it is collected by air light guides and transmitted to PMTs. The detector will be $10 \lambda_{i}$ deep and will be placed at $17 \mathrm{~m}$ from the interaction point to cover the pseudorapidity range $5.6 \lesssim \eta \lesssim 7.2$ where the baryon number density is expected to be large (Fig. 6).

It will search for events with large imbalance in electromagnetic and hadronic content and for abnormally penetrating objects.

It was originally designed and approved as a subsystem of the ALICE heavy ion experiment. Because of technical reasons it has been recently moved to the CMS experiment. Prototypes of the calorimeter have been constructed and tested with an electron beam at CERN. The last CASTOR calorimeter test took place in October 2001. The preliminary results and comparison with the NA52 and $\mathrm{H} 1$ calorimeters, employing similar technology are promising.

\section{Simulations of exotic events}

\subsection{Centauro events}

In order to illustrate the detector's sensitivity to new effects we have written the Monte-Carlo event generator CNGEN [22] embodying the described model, and used it to study the production of 


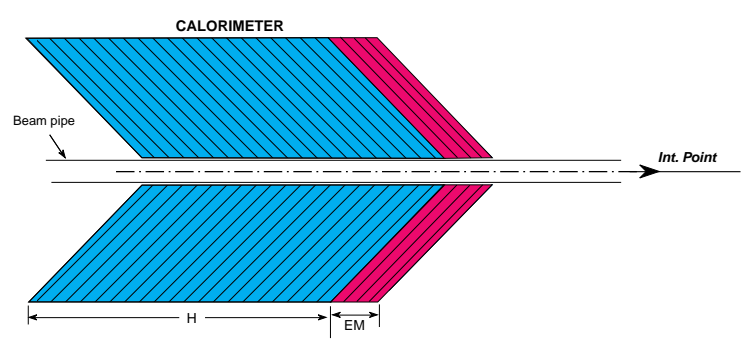

Figure 5.

Schematic side view of the CASTOR calorimeter.

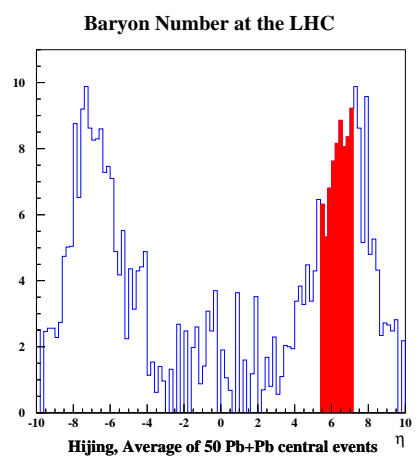

Figure 6.

Baryon number pseudorapidity distribution predicted by HIJING for central $\mathrm{Pb}+\mathrm{Pb}$ collisions at the LHC, the CASTOR acceptance is indicated.

Centauro in the forward, baryon-rich environment of a QGP fireball created in central $\mathrm{Pb}+\mathrm{Pb}$ collisions at the LHC, and the detector's response to them. The results of simulations of Centauro events formed in $\mathrm{Pb}+\mathrm{Pb}$ central collisions at $\sqrt{s}=5.5 \mathrm{~A} \mathrm{TeV}$ show that the characteristics of simulated Centauro events are apparently different from those obtained from conventional (e.g. HIJING) generators $[8,22,23]$.

In particular, Centauro events are characterized by almost total absence of the photonic component among secondary particles. The majority of secondary particles are baryons. Kaons which are emitted from the primary fireball can decay in principle into neutral pions which in turn give photons, but the overall neutral pion production is suppressed here strongly. Small multiplicity of Centauro events may be surprising for nucleus-nucleus collisions at such energies. It is illustrated in Fig. 7 which shows the average multiplicities of different kinds of particles produced via the Centauro mechanism and contained within the geometrical Castor acceptance. There are shown, as the examples, the average multiplicities for two sets of events characterized by a temperature $\mathrm{T}=$ $130,300 \mathrm{MeV}$ and a baryochemical potential $\mu_{b}=1.8,3.0 \mathrm{GeV}$ respectively, along with the HIJING prediction for comparison.
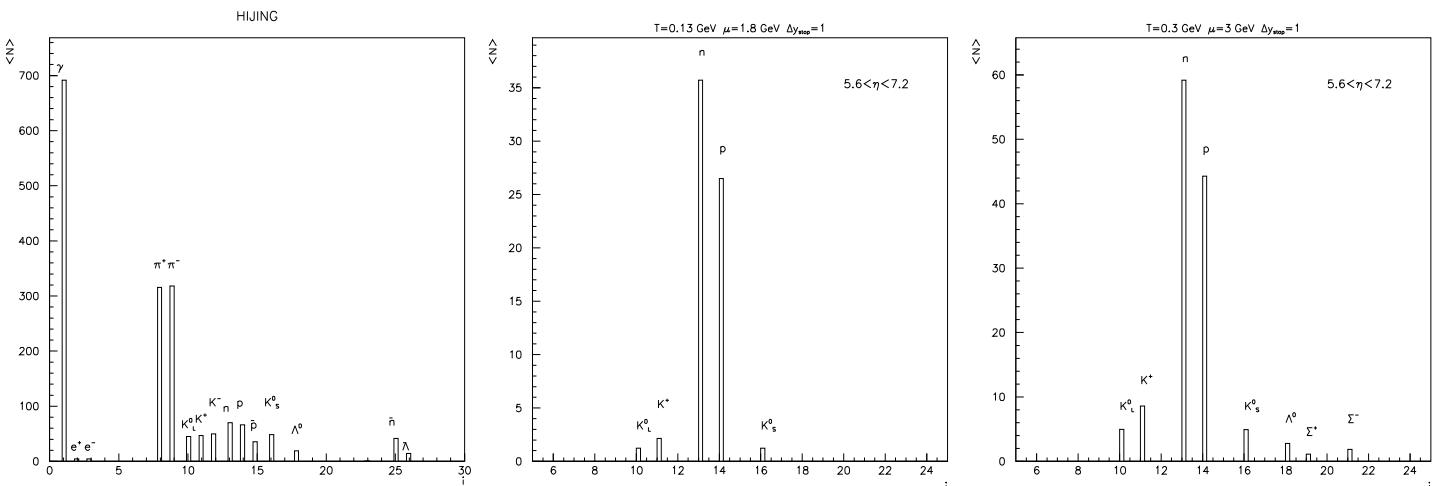

Figure 7. Average multiplicities of particles produced in conventional (HIJING) event and via Centauro mechanism. Only particles within CASTOR acceptance are shown.

Secondary particles in the Centauro events have larger mean transverse momentum in comparison with ordinary hadronic interactions. In conventional events the average transverse momentum of produced particles $\left\langle p_{T}\right\rangle=0.44 \mathrm{GeV} / \mathrm{c}$, as predicted by HIJING, which is several times smaller than that of Centauro events. Fig. 8 shows distributions of transverse momentum of strangelets and other Centauro decay products for three different temperatures $\mathrm{T}=130,200$ and $300 \mathrm{MeV}$. 

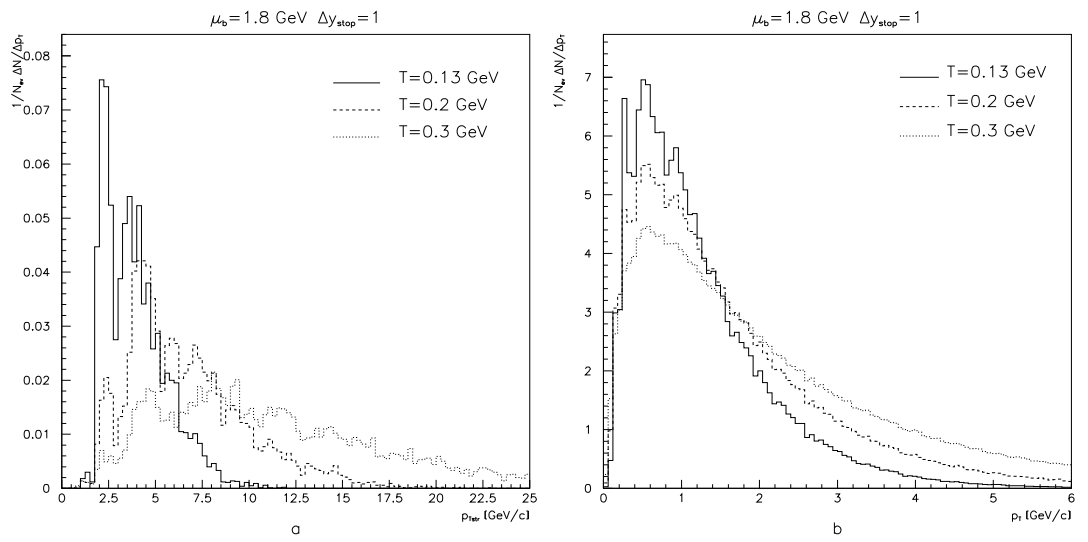

Figure 8. Transverse momentum distributions of (a) strangelets and (b) hadrons from Centauro decay.

The rapidity(pseudorapidity) distribution of decay products of the Centauro fireball depends mainly on the nuclear stopping power $\Delta y_{\text {stop }}$. Fig. 9 shows the distribution of particles from a decay of a simulated Centauro fireball with $\mathrm{T}=250 \mathrm{MeV}$ and $\mu_{b}=1.5 \mathrm{GeV}$. A significant fraction of them falls within the CASTOR acceptance, as well as $\simeq 25 \%$ of the associated strangelets.

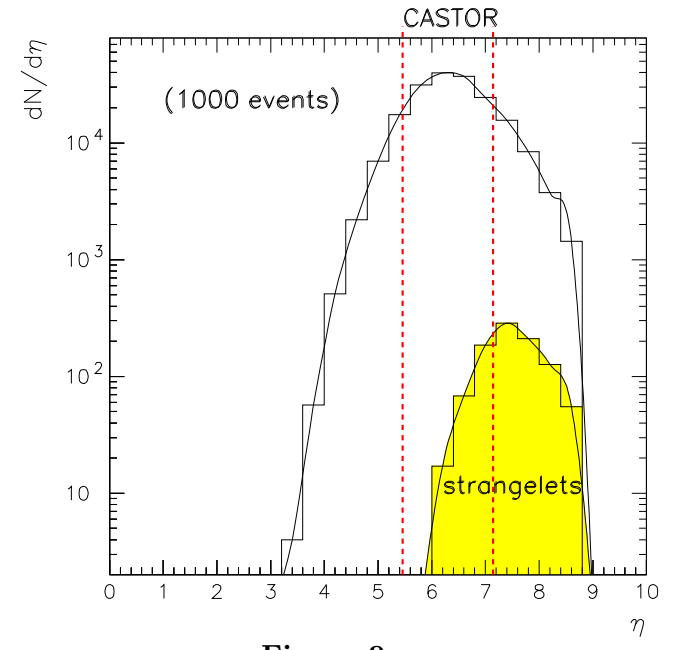

Figure 9.

Simulation of the production of Centauro and strangelets in central $\mathrm{Pb}+\mathrm{Pb}$ collisions at the LHC, the CASTOR acceptance is indicated.

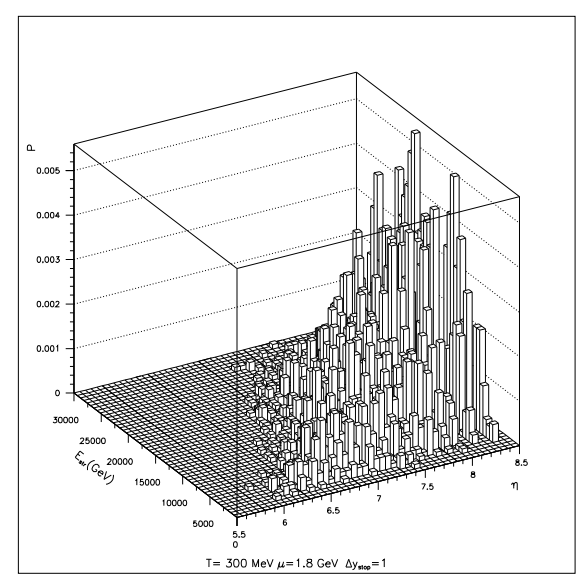

Figure 10.

Probability of a strangelet production as a function of its energy and pseudorapidity.

Fig. 10 shows two-dimensional lego histogram which illustrates the probability of a strangelet production, as a function of pseudorapidity and energy. Most of these would have $\mathrm{E}_{\text {str }}=5-10 \mathrm{TeV}$ and $\mathrm{A}_{s t r}=20-40$ [23] although strangelets with much higher energy are also expected to be produced.

\subsection{Exotic objects in deep calorimeters}

The important question is what signals will be produced by exotic objects during their passage through the deep calorimeter, and whether these signals can be distinguished from those produced by conventional events. A possible background was estimated by means of the HIJING generator. Three "exotic" scenarios were investigated. In the first one, it was assumed that strangelets were born according to some undefined mechanism anywhere among other conventionally produced particles [24]. 
In the second one, strangelets were considered to be the remnants of the Centauro fireball explosion. In this case the signal will be the sum of the strangelet signal and the signal produced by nucleons coming from the isotropic decay of the Centauro fireball [23]. In the third scenario it was assumed that fluctuations of electromagnetic to hadronic ratio were due to the production of a DCC cluster.

\subsubsection{Strangelets}

At the LHC kinematical conditions, the production of a variety of strangelets, characterized by a wide spectrum of the baryon number $\left(A_{\text {str }} \approx\right.$ several tens, temperature $T \approx 130-190 \mathrm{MeV}$ and quark chemical potential $\mu_{q} \approx 600-1000 \mathrm{MeV}$ ) should be possible. The scenario, in which strangelets are born anywhere among other conventionally produced particles was investigated in [24] for both short-lived and long-lived strangelets.

Unstable objects which can decay via strong interactions or the metastable ones decaying via weak nucleonic decays were named short-lived strangelets. If lifetimes of metastable strangelets are shorter than $\sim 10^{-10} \mathrm{~s}$ they could decay before reaching the CASTOR calorimeter and give the same picture as the unstable strangelets. The algorithm used in the calculations was the same as the one used previously for cosmic ray events. It was assumed that unstable strangelets decay very fast, practically at the point of their formation, thus the picture considered resolves into the simple case of a bundle of neutrons entering the calorimeter. The general conclusion concerning the signals produced in the CASTOR calorimeter by short-lived strangelets, formed in $\mathrm{Pb}+\mathrm{Pb}$ interactions at the $\mathrm{LHC}$ is the same as in the study of the cosmic-ray strangelets. Bundles of collimated neutrons can give in the CASTOR calorimeter an unconventional many-maxima signal.

The objects capable of reaching and passing through the CASTOR calorimeter without decay, i.e. having a lifetime $\tau_{0} \geq 10^{-8} \mathrm{~s}$ have been named long-lived strangelets. Similarly, as in the case of cosmic ray strangelets, the simplified picture [19] of the interaction of a stable strangelet in the calorimeter absorber was assumed.

The strangelet was considered as an object with the radius

$$
R=r_{0} A_{s t r}^{1 / 3}
$$

where the rescaled radius is

$$
r_{0}=\left(\frac{3 \pi}{2\left(1-\frac{2 \alpha_{c}}{\pi}\right)\left[\mu^{3}+\left(\mu^{2}-m^{2}\right)^{3 / 2}\right]}\right)^{1 / 3},
$$

$\mu$ and $m$ are the chemical potential and the mass of the strange quark respectively and $\alpha_{c}$ is the QCD coupling constant. The mean interaction path of strangelets in the tungsten absorber then is

$$
\lambda_{s-W}=\frac{A_{W} \cdot m_{N}}{\pi\left(1.12 A_{W}^{1 / 3}+r_{0} A_{s t r}^{1 / 3}\right)^{2}} .
$$

While penetrating through the calorimeter a strangelet collides with tungsten nuclei. At each collision the spectator part of the strangelet survives continuing its passage through the calorimeter while the wounded part is destroyed. Particles generated at the consecutive collision points interact with the tungsten nuclei in the usual way, resulting in the electromagnetic - nuclear cascade which developes in the calorimeter.

Penetration of stable strangelets through the calorimeter was simulated, assuming $\alpha_{s}=0.3$ and several different sets of the initial strangelet parameters: $\left(\mu_{q}=300,600,1000 \mathrm{MeV} ; A_{s t r}=15\right.$, 20,$40 ; E_{s t r} \approx 8-40 \mathrm{TeV}$ (or $400-1000 \times \mathrm{A} \mathrm{GeV}$ )). Examples of transition curves produced in the CASTOR calorimeter by various stable strangelets are presented in Fig. 11. The curves are limited to the one calorimeter octant which contains the strangelet. The strangelet cascade profiles are compared with those of the conventional background, produced by particles generated by HIJING (full line histogram), after the subtraction of the energy carried by the strangelet. It can been concluded that stable strangelets can produce in the calorimeter long range many-maxima cascades, manifestly different from these produced by a conventional event. 

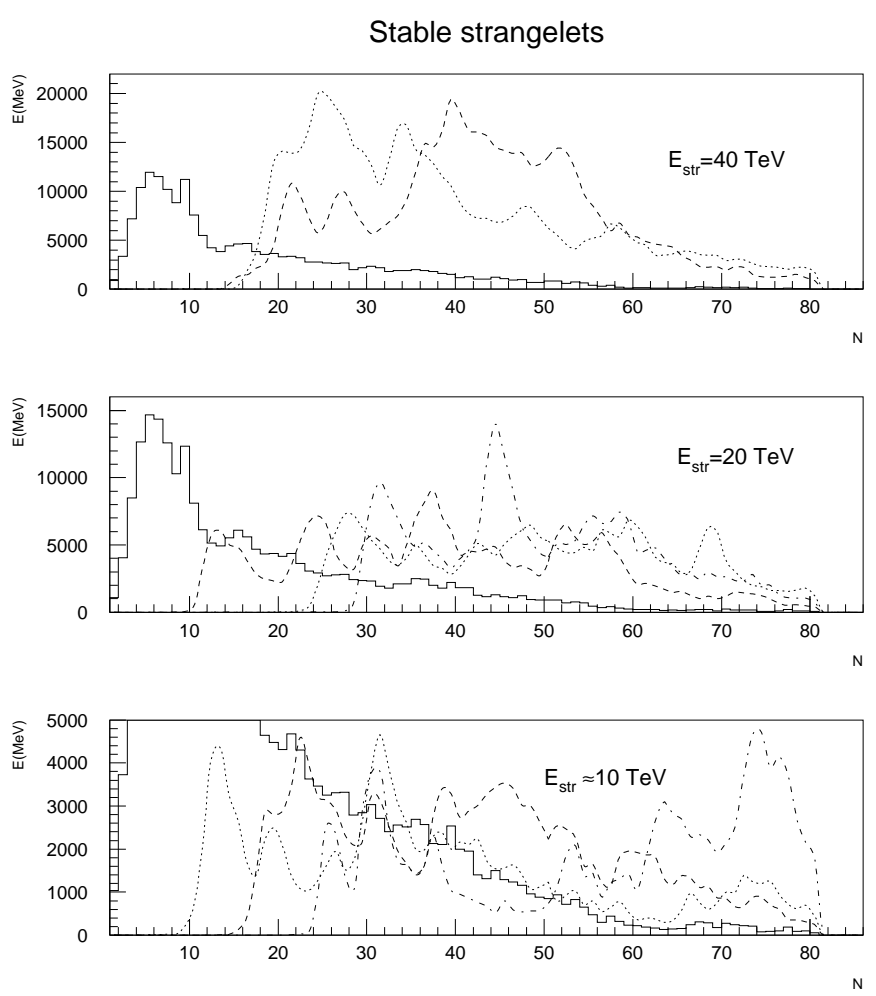

Figure 11. Transition curves of stable strangelets with energy $E_{\text {str }}=10-40 \mathrm{TeV}$, baryon number $A_{s t r}=15-40$, quark chemical potential $\mu_{q}=600,1000 \mathrm{MeV}$. Energy deposit $(\mathrm{MeV})$ in the calorimeter layers, in the octant containing a strangelet, is shown. Full line histograms show the HIJING estimated background.

\subsubsection{Strangelets and Centauros}

The other question concerns the shape of transition curves produced in the calorimeter by:

- strangelets born in the Centauro fireball explosion and registered in the apparatus together with other Centauro decay products,

- or Centauro fireball decay products without accompanying strangelets emission.

Exotic events generated by means of the Centauro code were passed through the CASTOR calorimeter, by using a modified version of GEANT $3.21[8,23]$. For each event, the transition curves produced by the Centauro fireball decay products, by the accompanying strangelet, and by background of conventionally produced particles, were simulated separately. All these three contributions separately and also their sum, constituting the so-called "mixed" event, were compared with the conventional transition curve, produced by HIJING. Centauro events characterized by various values of parameters: temperature $(T=250,300 \mathrm{MeV})$, quarkchemical potential $\left(\mu_{q}=600,1000\right.$ $\mathrm{MeV})$ and nuclear stopping power $\left(\Delta y_{\text {stop }}=0.5,1.0,1.5\right.$ corresponding to an effective stopping in the range $\sim 1.5-3.0$ pseudorapidity units) were analysed. In these events strangelets with baryonic numbers $A_{\text {str }}=20-40$ and energies $E_{\text {str }} \simeq 8-20 \mathrm{TeV}$ were formed. Two examples of the resulting transition curves produced by a Centauro event with an unstable and stable strangelet born among its secondaries are shown in Fig. 12. The energies of exotic objects (Centauro and the strangelet) within the CASTOR acceptance were: $\sim 128 \mathrm{TeV}$ and $\sim 126 \mathrm{TeV}$ respectively in comparison to $\sim$ $156 \mathrm{TeV}$ predicted by HIJING for the conventional central $\mathrm{Pb}+\mathrm{Pb}$ event.

Our analysis indicates that the Centauro events (whether accompanied and not accompanied by a strangelet) can be easily distinguished from conventional events. Centauro transition curve in the calorimeter is expected to have manifestly different shape and longer extent than that produced by a 

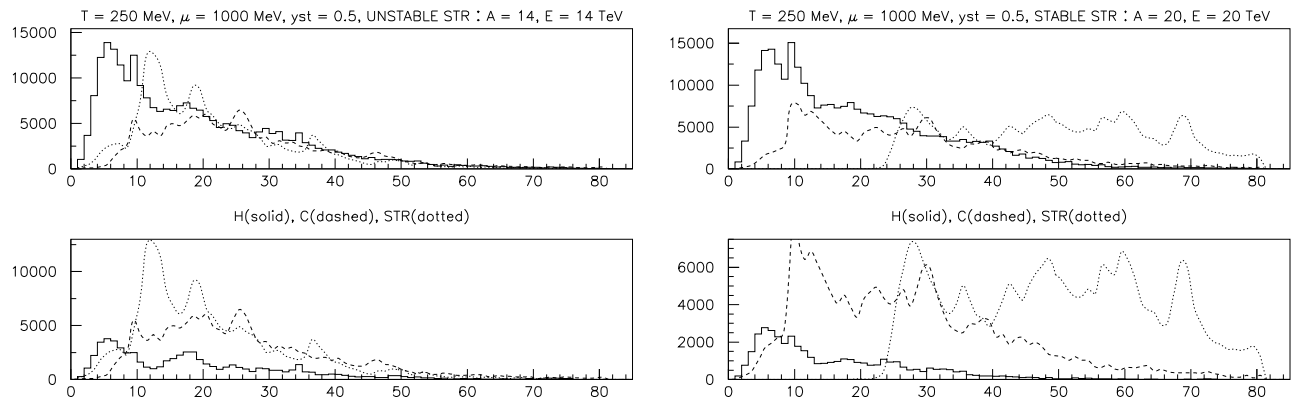

Hback(solid), C(dashed), STR(dotted)

Hback(solid), C(dashed), STR(dotted)
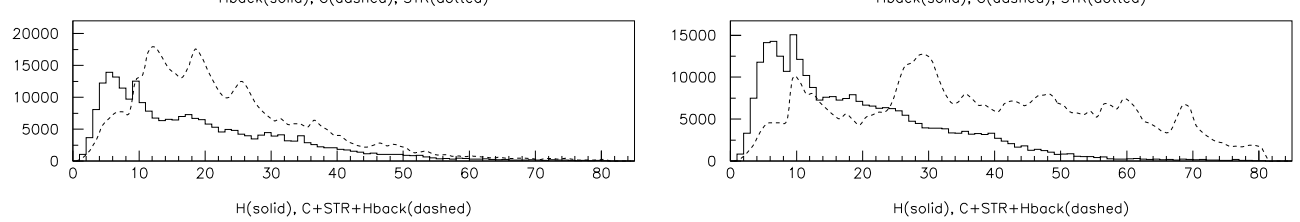

Figure 12. Transition curves produced by Centauro event "C"with unstable or stable strangelets "STR", in comparison with HIJING "H". Energy deposit (MeV) in consecutive calorimeter layers, in the octant containing a strangelet, is shown.

conventional event. The Centauro produced signal has a maximum at about 14th calorimeter layer with the average at $\left\langle N_{\text {Cent }}\right\rangle \simeq 25$. The HIJING event produces the maximum of the signal at about the 8th calorimeter layer, with the average at $\left\langle N_{H I J}\right\rangle \simeq 19$. Generally, the Centauro produced signal is stronger in the deeper (hadronic) part of the calorimeter, as opposed to the HIJING generated one, which is peaked in the electromagnetic section of the calorimeter.

\subsection{3. $D C C$}

The QCD phase transition from normal hadronic matter to the Quark-Gluon-Plasma, manifests itself in two forms: deconfinement transition and chiral symmetry restoration. One of interesting consequences of the chiral transition is the possible formation of a chiral condensate in an extended domain, such that the direction of the condensate is misaligned from the true vacuum direction. Formation of these so called Disoriented Chiral Condensate (DCC) domains in high energy collisions of both hadrons and heavy ions, has been proposed by many authors (see for example [45]). A basic signature of DCC production is the presence of very large event-by-event fluctuations in the fraction of produced neutral pions. Thus, there are some suspicions that this phenomenon could also be responsible for the Centauro-like events. We have simulated the passage of DCC clusters (both charged and neutral) through the deep calorimeter and they also show characteristic transition curves (Fig. 13).

\subsubsection{Summary of exotic events}

In Fig. 13 are shown for comparison examples of transition curves produced by different exotic phenomena, among them charged and neutral DCC clusters, strangelets, Centauros and mixed events. The curves presented are the sum of the exotic signals and the conventional background. All these phenomena give different patterns and could be easily distinguished from conventional events as well as from each other. This method is sensitive to the detection of any kind of strangelets: both neutral and charged, as well as short-lived and long-lived. This is an important feature in the light of present experiments which are only able to detect strangelets with lifetimes longer than $\sim 10^{-9} \mathrm{~s}$. It is seen that the energy deposition pattern in a deep calorimeter can be an excellent signature of a QGP state decaying in different exotic ways.

For the configuration of the calorimeter studied here the extraction of the exotic phenomena signal from the conventionally produced background will be straight forward for energies higher that $\sim 10 \mathrm{TeV}$. In order to study the sensitivity of the calorimeter to abnormally penetrating objects 

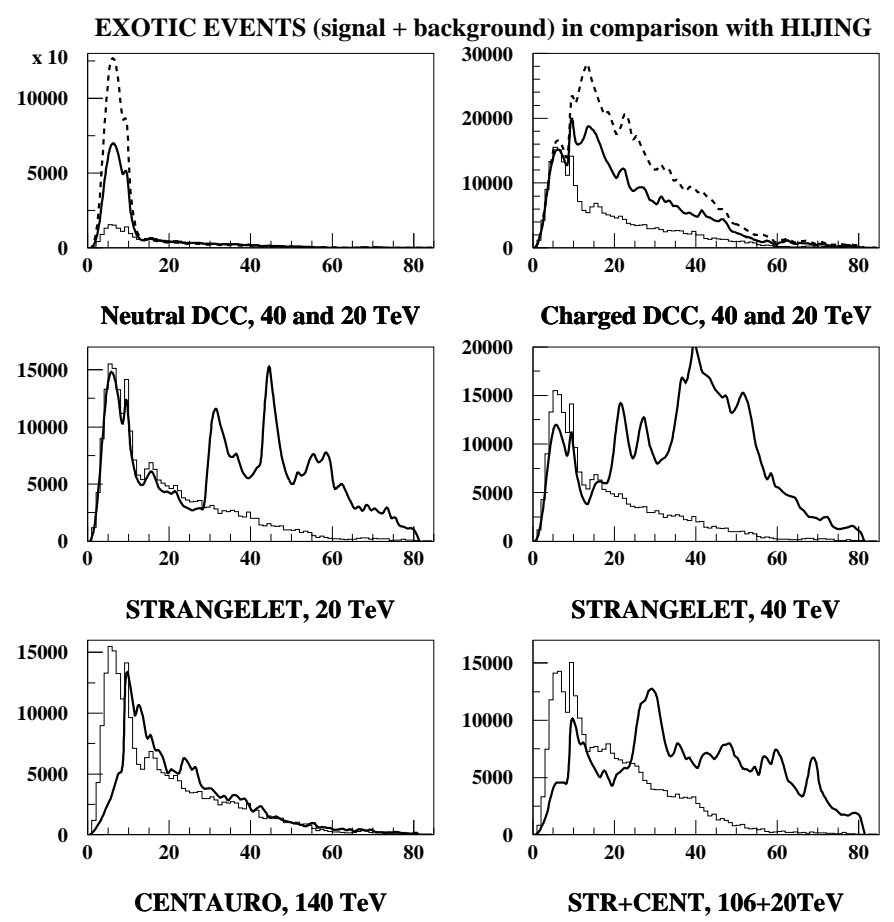

Figure 13. Simulated signals produced by different kinds of events. The deposit of energy in one sector (hit by the exotic object) vs. layer number of the calorimeter is shown.

Thin continuous line corresponds to the HIJING event.

of lower energies, we developed a neural network technique [25] based on a multilayer perception network. On the input layer are fed the signals from the readout units of one octant, the output layer consists of one neuron providing a yes/no answer, and there is one hidden layer. We tested the method using simulated strangelets. One set of $100005 \mathrm{TeV}$ strangelets and 10000 background events was used as the training sample and a different such set as the evaluation sample. Figure 14 shows the enhancement obtained in the signal/background ratio through the application of this technique to a calorimeter consisting of 15 readout units per octant, for different numbers of neurons in the hidden layer. An enhancement of $O\left(10^{4}\right)$ is obtained for efficiency $\simeq 95 \%$.

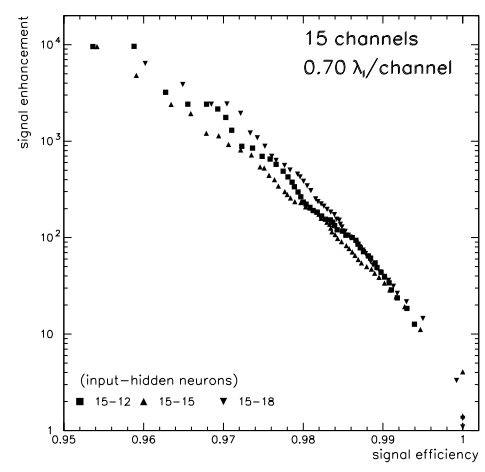

Figure 14. Enhancement of the signal/background ratio for strangelet detection obtained through application of the neural network technique.

This work was partly supported by Polish State Committee for Scientific Research grant No. 2P03B 01118 and SPUB-M/CERN/P-03/DZ 327/2000.

\section{References}

[1] Z. Fodor and S.D. Katz, hep-lat/0106002

[2] J. Berges and K. Rajagopal, hep-ph/9804233 (1998);

R. Rapp, T. Schaefer, E. V. Shuryak and M. Velovsky, hep-ph/9711396 (1997).

[3] R.M. Nikolaeva, V.A. Nikolaev and O.G. Tkachev, J. Phys. G: Nucl. Part. Phys.18(1992) 1149; S.M.H.Wong, hep-ph/0202250. 
[4] C. Greiner et al., Phys. Rev. D38 (1988) 2797.

[5] V. I. Yakovlev, Proc. of the VIIth Intern. Symp. on VHECRI, Ann Arbor (1992), ed. L. Jones, 1993, p. 154; J. M. Dremin et al., Voprosy atomnoy nauki i tekhniki, seria: Tekhnika fizitcskeskovo eksperimenta, Vypusk 4(25), p. 3, 1985; ISSN-0207-0480.

[6] G. Altarelli et al., Z. Phys. C45 (1989) 109; A. B. Kurepin, ALICE/95-47, Internal Note/PHY 1995.

[7] C.M.G. Lattes, Y. Fugimoto and S. Hasegawa Phys. Rep. 65 (1980) 151;

S. Hasegawa, Proc. VIII Intern. Symp. on VHECRI, Tokyo, 1994, ed. by Local Organising Commitee, p. 115.

[8] E. Gładysz-Dziaduś hep-ph/0111163, to be publ. in Phys. Part. Nucl. Vol.34, \#1 (2003).

[9] T. Arisawa et al., Nucl. Phys.B424 (1994) 241.

[10] Z. Buja et al., Proc. 17th ICRC (Paris) vol. 11 p 104 (1981).

[11] L. T. Baradzei, Nucl. Phys. B370 (1992) 365.

[12] M. Tamada Inst. Cosmic Ray Research, Univ. Tokyo ICRR-Report-454-99-12; M. Tamada and A. Ohsawa, Nucl. Phys. B581 (2000) 73.

[13] J. D. Bjorken and L. D. McLerran, Phys. Rev. D20 (1979) 2353.

[14] K. Goulianos, Comments Nucl. Part. Phys. 17 (1987) 195.

[15] A.D. Panagiotou et al., Phys. Rev. D45 (1992) 3134; A.D. Panagiotou et al., Z. Phys. A333 (1989) 355.

[16] M.N. Asprouli, A.D. Panagiotou and E. Gładysz-Dziaduś Astrop. Phys. 2 (1994) 167; E. Gładysz-Dziaduś and A.D. Panagiotou, Proc. Int. Symp. on Strangeness \& Quark Matter (Krete) eds Vassiliadis G et al World Scientific p 265 (1995).

[17] C.E. Navia et al., Phys. Rev. D55 (1997) 5834.

[18] K. Kang and A. White, Phys. Rev. D42 (1990) 835.

[19] E. Gładysz-Dziaduś and Z. Włodarczyk J. Phys. G: Nucl. Part. Phys. 23 (1997) 2057.

[20] J. Bartke, E. Gładysz-Dziaduś, M. Kowalski and A. D. Panagiotou, ALICE Internal Note, PHYS/93-12.

[21] A. Angelis et al., CASTOR draft proposal, ALICE/97-07, Internal Note/CAS; A.L.S. Angelis and A.D. Panagiotou J. Phys. G: Nucl. Part. Phys. 23 (1997) 2069; A.L.S. Angelis et al., Nuovo Cim. 24C (2001) 755;

A.L.S. Angelis et al., Nucl. Phys. Proc. Suppl. 97 (2001) 227.

[22] E. Gładysz-Dziaduś et al., Internal Note ALICE-INT-1997-06.

[23] E. Gładysz-Dziaduś, Internal Note ALICE-INT-2001-21;

M. Sowa, Master Degree Thesis, Institute of Nuclear Physics, Kraków (Poland), 2000.

[24] A. L.S. Angelis, J. Bartke, E. Gładysz-Dziaduś and Z. Włodarczyk, EPJ direct C9 (2000) 1.

[25] A.L.S. Angelis et al., 6th Intern. Conf. on Strange Quarks in Matter, Frankfurt, Germany, 2001, to be publ. in J. Phys. G: Nucl. Part. Phys.;

G. Mavromanolakis $2001 \mathrm{PhD}$ thesis, University of Athens, ALICE note in preparation.

[26] O.P. Theodoratou and A.D. Panagiotou Astrop. Phys. 13 (2000) 173.

[27] M. S. Desai and G. L. Shaw, AIP Conf. Proc. No. 340 (1995) 53.

[28] A. Dar, A. de Rújula and U. Heinz, CERN-TH/99-324; hep-ph/9910471.

[29] W. Busza et al., hep-ph/9910333, 1999;

R. L. Jaffe, W. Busza, J.Sandweiss and F. Wilczek, Rev. Mod. Phys. (in press) 2000.

[30] J. Madsen, hep-ph/0008217, 2000, Phys. Rev. Lett.85 (2000) 4687.

[31] J. Madsen, e-print, hep-ph/0112153.

[32] E. Witten, Phys. Rev. D30 (1984) 272.

[33] J. Madsen, "Physics and Astrophysics of Strange Quark Matter", in "Hadrons in Dense Matter and Hadrosynthesis", Cape Town (1998), Lecture Notes in Physics, Springer, eds. J. Cleymans et al., p. 162; eprint: astro-ph/9809032;

C. Greiner and J. Schaffner-Bielich, "Physics of Strange Matter" in "Heavy Elements and Related New Phenomena", ed. R. K. Gupta and W. Greiner, (Singapore: World Scientific); preprint LBNL-41324 and eprint: nucl-th/9801062;

F. Weber, J. Phys.G: Nucl. Part. Phys. 25 (1999) R195.

[34] A. Baltz et al., Phys. Lett. B325 (1994) 7.

[35] R. Klingenberg, J. Phys. G: Nucl. Part. Phys. 25 (1999) R273.

[36] S. Kabana for the NA52 Coll., G. Ambrosini et al., J. Phys. G: Nucl. Part. Phys. 23 (1997) 2135.

[37] D.P. Anderson, E.T. Herrin, V. L. Teplitz and I. M. Tibuleac, eprint astro-ph/0205089.

[38] M. Rybczyński, Z. Włodarczyk and G. Wilk, hep-ph/0109225.

[39] T. Saito, Y. Hatano, Y. Fukada and H. Oda, Phys. Rev. Lett. 65 (1990) 2094.

[40] P. B. Price et al., Phys. Rev. D18 (1978) 1382.

[41] M. Ichimura et al., Nuovo Cimento A106 (1993) 843.

[42] CosmoLEP Report 1, CERN LEPC 95-5, 1999, CERN EP/2000-152.

[43] V. A. Antonova et al., Nucl. Phys. B (Proc. Suppl.) 75 A (1999) 333.

[44] S. B. Shaulov, Heavy Ion Physics 4 (1996) 403;

G.Wilk and Z.Włodarczyk, Heavy Ion Physics.

[45] J. D. Bjorken, Acta Physica Polonica B28 (1997) 2773. 4 (1996) 395. 